\title{
Milliarcsecond Structure of Microarcsecond Sources
}

\section{Roopesh Ojha*t}

United States Naval Observatory/NVI, 3450 Massachusetts Ave NW, Washington DC 20392

E-mail: rojha@usno.navy.mil

\section{T. Joseph W. Lazio}

Remote Sensing Division, Naval Research Lab, Code 7213, Washington, DC 20375-5351

E-mail: lazio@nrl.navy.mil

\author{
Alan L. Fey \\ United States Naval Observatory, 3450 Massachusetts Ave NW, Washington DC 20392 \\ E-mail: afey@usno.navy.mil
}

\section{David L. Jauncey}

Australia Telescope National Facility CSIRO, P.O. Box 76, Epping, NSW 1710, Australia

E-mail: David.Jauncey@csiro.au

\author{
James E. J. Lovell \\ School of Mathematics \& Physics, Private Bag 21, U. of Tasmania Hobart TAS 7001, Australia \\ E-mail: Jim.Lovelleutas.edu.au
}

\section{Kenneth J. Johnston}

United States Naval Observatory/NVI, 3450 Massachusetts Ave NW, Washington DC 20392

E-mail: kjj@usno.navy.mil

We present the key findings of an ongoing program of Very Long Baseline Interferometry (VLBI) observations of scintillating and non-scintillating flat-spectrum extragalactic radio sources whose scintillation status was determined by the Micro-Arcsecond Scintillation-Induced Variability (MASIV) survey. Both single frequency $(8.4 \mathrm{GHz})$ observations to compare morphologies, and multi-frequency observations (from $330 \mathrm{MHz}$ through $22 \mathrm{GHz}$ ) to study scattering behaviour have been carried out. Though scintillation probes the structure of quasars on scales of tens of microarcseconds, information at the milliarcsecond scale probed by these VLBI observations is proving invaluable in furthering our understanding of this phenomenon. Also, the common occurence of scintillation in compact radio sources might be the ultimate limitation for VLBI astrometry (which among other things defines the International Celestial Reference Frame) in the sense that the final precision of reference frame source positions are likely to be influenced by the presence of scintillation.

Bursts, Pulses and Flickering:Wide-field monitoring of the dynamic radio sky June 12-15 2007 Kerastari, Tripolis, Greece

\footnotetext{
*Speaker.

$\dagger$ I acknowledge the support of the American Astronomical Society and the National Science Foundation in the form of an International Travel Grant, which enabled me to attend this conference.

${ }^{\ddagger}$ Basic research in radio astronomy at NRL is supported by NRL 6.1 Base funding.
} 


\section{Introduction}

Interstellar scintillation (ISS, [11]) has been shown to be the principal cause of the rapid fluctuations in flux density seen in many compact, flat-spectrum radio sources at centimeter wavelengths. The predicted time delay in the arrival of the variability pattern at two widely separated telescopes has been observed [1]. Such time delay measurements allow measurement of the transverse component of the interstellar medium (ISM) velocity in the direction of the baseline formed by the two telescopes. Further, an annual cycle in the characteristics of the variability over the course of a year has been discovered for several sources [3]. When the Earth is moving in the same direction as the ISM the relative speed is small and the variations are slow. Six months later the Earth is moving in the opposite direction so the relative speed is high and the variations are more rapid. Finally, the MASIV (Microarcsecond Scintillation-induced Variability) survey [5], [6] has established a Galactic latitude dependence in the distribution of scintillators with fewer scintillators at high Galactic latitudes at the $98 \%$ confidence level. The only plausible explanation for these results is ISS in the ionized, turbulent ISM of our Galaxy.

The MASIV survey is a large-scale VLA survey at $5 \mathrm{GHz}$ that was undertaken to construct a statistically significant sample of sources which are intraday variable (IDV, [12]). Such a sample is essential to address a number of astrophysical questions by examining the microarcsecond structure accessed by IDV and by probing the turbulent ionized ISM responsible for the scintillation. The survey comprised four 72 hour epochs in January, May, September 2002 and January 2003, during VLA reconfiguration. A fifth epoch has since been observed in January 2006. The VLA was divided into 5 sub-arrays of 5 or 6 antennas each. An initial sample of 550 compact, flat spectrum sources were observed at $5 \mathrm{GHz}$ with 60 seconds on-source per scan and about 6 scans per source per day. There were a total of 10000 scans per epoch. After removing sources with structure or confusion, the final sample contained 482 sources. Once false positives were accounted for, the fraction of scintillators was $268 / 482=56 \%$. Thus IDV is a widespread phenomenon among flatspectrum active galactic nuclei (AGN). However, rapid, large-amplitude variables such as $J 1819+$ 3845 [2] remain uncommon.

Here we discuss past and ongoing studies of the properties of scintillators and non-scintillators at the milliarcsecond scales of Very Long Baseline Interferometry (VLBI).

\section{Milliarcsecond Scale Structure of Scintillating Sources}

An early result from MASIV was an observed increase in the fraction of highly variable scintillators (those with rms flux density variations above 4\%) with decreasing flux density. Also seen was an increase in their fractional amplitude of variability with decreasing flux density. These results suggested that the weaker sources may be more "core-dominated" (more precisely, less milliarcsecond "jet" dominated). Thus, low flux density scintillating sources may form a distinct population with a morphology that differs from that of non-scintillators.

We used the Very Long Baseline Array (VLBA) operated by the National Radio Astronomy Observatory (NRAO) ${ }^{1}$ to image the milliarcsecond-scale structure of a sample of 75 low flux

\footnotetext{
${ }^{1}$ The National Radio Astronomy Observatory is a facility of the National Science Foundation operated under cooperative agreement by Associated Universities, Inc.
} 
density scintillator (hereafter LFS) sources discovered in the MASIV survey [8]. These sources had a mean and median flux density of $120 \mathrm{mJy}$ and $110 \mathrm{mJy}$, respectively. Each source was observed in 4 scans of 7.5 minutes each in full polarization mode at $8.4 \mathrm{GHz}$. The data processing was done in a standard fashion using NRAO's AIPS package and the Caltech Difmap package. The final self-calibrated total intensity visibility data were modelfit to characterize the images.

Even a cursory examination of the images immediately reveals that most are almost unresolved or have a single compact component (see Fig 1, [8]). A more quantitative analysis confirms that on a number of measures (described below) scintillating extragalactic radio sources in this sample are among the most compact and core dominated of all extragalactic radio sources. In fact 19 of these 75 sources (25\%, an unusually high fraction) have completely unresolved cores. A typical image of a LFS is shown in Fig 1.

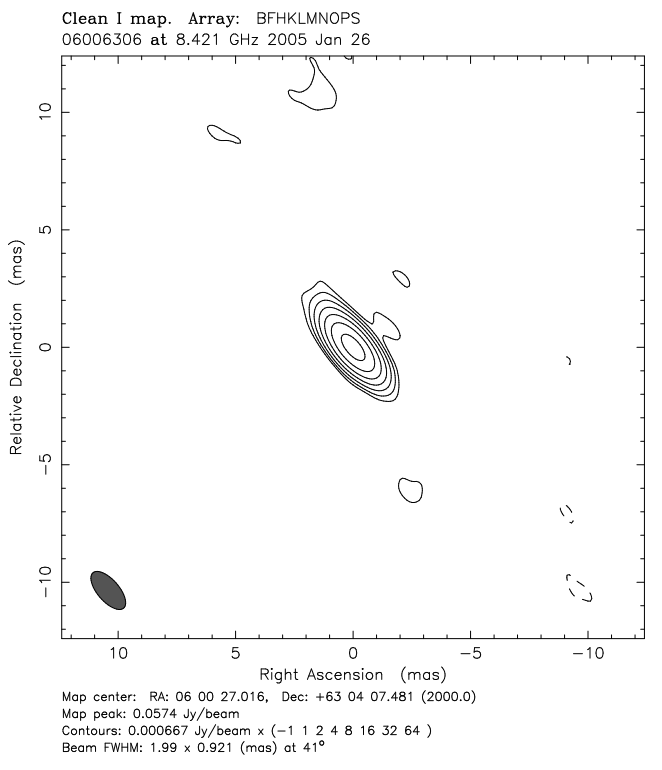

Figure 1: Typical morphology of a Low Flux Density Scintillator

Having constructed the LFS sample we compared their milliarcsecond morphology to a sample of high flux density scintillators and high flux density non-scintillators, hereafter HFS and HFN respectively [9]. Broadly, we sought to address the following two questions:

Are there any morphological differences, at mas scales, between high flux density, $\sim 1 \mathrm{Jy}$, and low flux density, $\sim 0.1 \mathrm{Jy}$, scintillating sources?

Are there any morphological differences, at mas scales, between scintillating and non-scintillating sources?

The three samples used for this comparative study are well-defined and uniformly selected. All sources are point sources at all resolutions of the VLA, have a spectral index flatter than 0.5 (from 1.4 to $4.9 \mathrm{GHz}$ ) and they were all observed in the $8.4 \mathrm{GHz}$ band with the VLBA. The two scintillating samples were found to be scintillating (by the MASIV survey) with rms flux density variations during a 72 hour period of $S_{r m s}>\left[(0.003)^{2}+(0.02 S)^{2}\right]^{1 / 2} \mathrm{Jy}$, where the first term represents the uncertainty in flux density measurements due to noise and confusion while the second term represents uncertainty proportional to flux density that arises mostly from pointing offsets [6]. 
The LFS sample has 75 sources with flux density ranging from 50 to $370 \mathrm{mJy}$ obtained from our VLBA observations as described above. The HFS sample consists of 18 scintillators with flux density $\sim 1 \mathrm{Jy}$. Structural information for these sources was obtained from the U S Naval Observatory (USNO) Radio Reference Frame Image Database ${ }^{2}$ (RRFID). This is a database of images of all radio reference frame sources at the same wavelengths as those used for precision astrometry. The RRFID also contained data for 144 high flux density $\sim 1$ Jy non-scintillating sources. These make up our third, HFN, sample. The two high flux density samples have comparable flux density distributions and both have distinctly higher flux density distributions than the low flux density sample.

Three different measures were used to compare the morphologies of these three samples in a quantitative manner: core fraction, flux-weighted radial extent and unweighted radial extent. The core fraction is essentially an estimate of the core dominance of these sources and is defined as the ratio of core flux density to total flux density, $C=\sum_{i}\left(S_{i}\right)_{\text {beam }} / \sum_{i} S_{i}$, where $S_{i}$ is the flux density of the $i$ th CLEAN component. Core flux density is defined as the sum of the CLEAN-ed flux density within one synthesized beam and total flux density is the total CLEAN-ed flux density i.e. the sum of all CLEAN components. On a Kolmogorov-Smirnov (K-S) test, a common core fraction population for LFS (HFS) and HFN sources is rejected at the 99\% (95\%) level. Thus both samples of scintillating sources have significantly higher core fractions than the non-scintillating sources.

The flux-weighted radial extent is a complementary measure of core dominance, which is defined as, $R=\sum_{i} S_{i} r_{i} / \sum_{i} S_{i}$, where $r_{i}$ is the radius at which the $i$ th CLEAN component has flux density $\mathrm{Si}$. Here $R$ has units of mas. A K-S test rejects a common parent population for LFS (HFS) and HFN sources at the $99 \%$ (99\%) while both scintillating samples have a $68 \%$ probability of being derived from the same core fraction population.

The unweighted radial extent is a straightforward measure of overall source size i.e. the maximum radial extent of the source. It is the angular radius that contains $95 \%$ of the total CLEAN-ed flux density. We first calculate a quantity $\mathrm{E}$, similar to $\mathrm{C}$ above and defined as, $E=\sum_{i}\left(S_{i}\right)_{\theta} / \sum_{i} S_{i}$, where the numerator represents the flux density contained within an area of angular radius $0 \leq \theta<\infty$. The maximum radial extent is then defined as the value of $\theta$ when $E \geq 0.95$. Here $\theta_{0.95}$ has units of milliarcseconds. Once again, a K-S test rejects a common parent population for LFS (HFS) and HFN sources at the 99\% (97\%) level. The LFS and HFS samples have a $50 \%$ probability of being derived from a common parent core fraction population.

Thus all the metrics we used lead us to conclude that both low and high flux density scintillators have significantly different morphologies than non-scintillators with a higher proportion of their flux in a compact core and a smaller overall angular size. This leads to the important conclusion that the scintillating component lies in the core.

Low and high flux density scintillators do not have significantly different morphologies and their morphological properties are consistent with their sharing the same parent core-fraction population.

There are two important ways the above conclusions can be made more robust. One is to increase the size of the rather modest-sized HFS sample which has only 18 sources. However, this is large enough for the K-S test to be valid. A more serious concern is that in the comparison of LFS

\footnotetext{
${ }^{2}$ Available at http://www.usno.navy.mil/RRFID
} 
sources with HFN sources, sensitivity limits could bias the results as weak extended structure in the LFS sample might not be detected. This potential bias can be addressed by creating a low flux density non-scintillating (LFN) sample to compare with the LFS sample. Observations to create this LFN sample have been made and calibrated and analysis is in progress.

\section{Scatter broadening of Scintillating and Non-Scintillating AGNs}

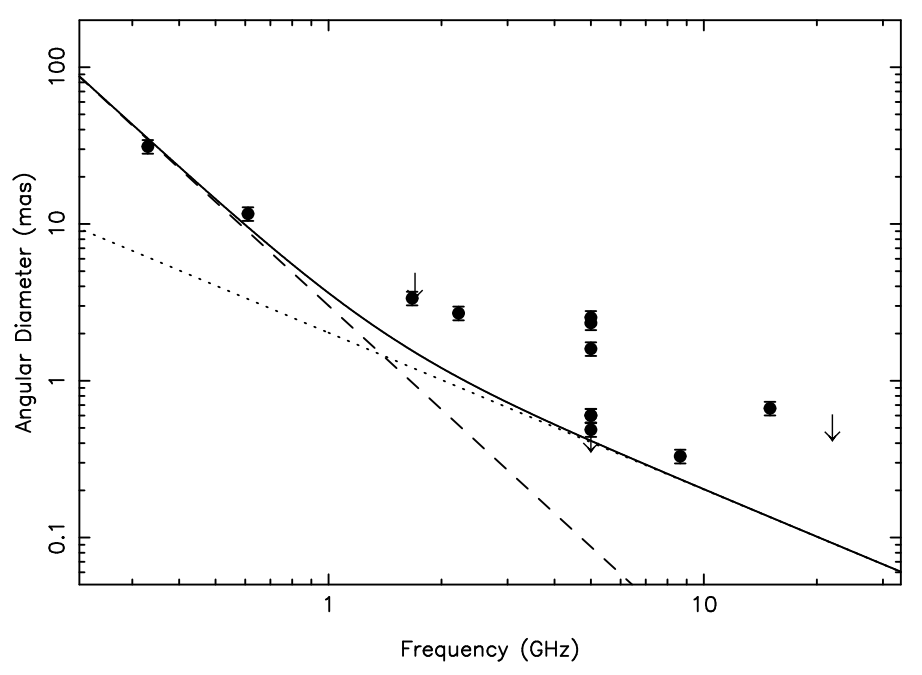

Figure 2: Fitting result for the source J2022 +6136 for which a relatively large scattering diameter, 3.1 mas at $1 \mathrm{GHz}$, is inferred. Solid circles show measured diameters, arrows indicate upper limits, the solid line indicates the fit of the equation to the observations, the dashed line indicates the inferred scattering diameter and the dotted line indicates the inferred intrinsic diameter. Uncertainties on the angular diameters are plotted, but in many cases are comparable to the size of the symbol.

We have made multi-wavelength observations to provide a statistically significant uniform data set to examine the core structure of extremely compact AGN, to study their scattering properties and to search for indications of an intergalactic medium. VLBA observations of 58 compact extragalactic sources were made at $0.33,0.61,1.6,2.3$ and $8.4 \mathrm{GHz}$ [10]. Approximately $75 \%$ of these objects display IDV. With archival images for some of these sources from the RRFID at 22 and 43 $\mathrm{GHz}$ we have information on up to 7 frequencies. All sources have angular diameter measurements for at least three frequencies. Gaussian component models were fit to the final calibrated visibility data. Where more than one component was required, the most compact component was identified as the "core", as the most compact component will be the one for which scattering effects will be most apparent. For the few sources where the most compact component was not also the brightest at all frequencies, the compact and bright component that could be consistently identified as the same at all frequencies was taken to be the "core".

One effect of angular broadening is that the observed angular diameter scales as $\lambda^{2.2}$. We fit the measured angular diameters to $\theta^{2}=\left(\theta_{s} v^{-2.2}\right)^{2}+\left(\theta_{i} v^{x}\right)^{2}$, where $\theta_{s}$ and $\theta_{i}$ are the scattering and intrinsic (FWHM) diameters of the AGN, respectively, at the fiducial frequency of $1 \mathrm{GHz}$. After considering both $x=0$ (frequency-independent intrinsic diameter, for a flat spectrum source) and $x=-1$ (frequency scaling for a single incoherent synchrotron component), the value of $x$ that 
produced the lower $\chi^{2}$ was selected. An example of a fit to a strongly scattered source is shown in Fig 2. The results of this analysis are presented in [4]. We briefly report some key results below.

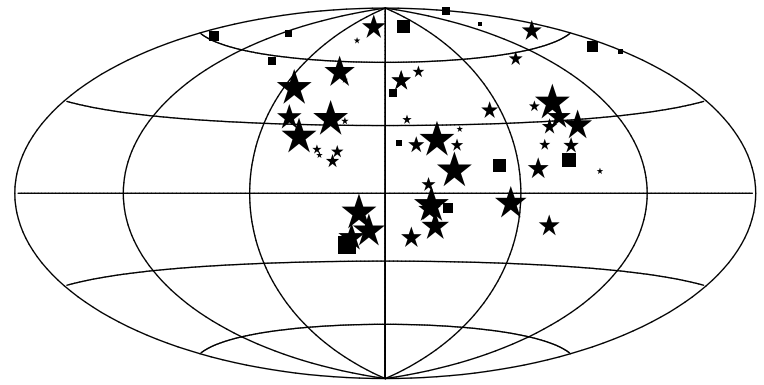

Figure 3: The distribution of sources observed as a function of Galactic coordinates. The Galactic anticenter is at the center of the plot, and longitude increases to the left. Stars indicate sources that scintillate; squares indicate non-scintillating sources. The size of the symbol is qualitatively proportional to the inferred scattering diameter.

Figure 3 shows the distribution of sources observed as a function of Galactic coordinates. In contrast to earlier work that shows interstellar scattering increasing rapidly at low latitudes, there is no statistically significant correlation of the inferred scattering diameter with either (the absolute value of) Galactic latitude $b$ or with ecliptic latitude $\beta$, nor with $\cos (|b|)$ and $\cos (|\beta|)$. There is also no correlation between inferred scattering diameter and solar elongation. We attribute this to having few, essentially no, lines of sight into the inner Galaxy.

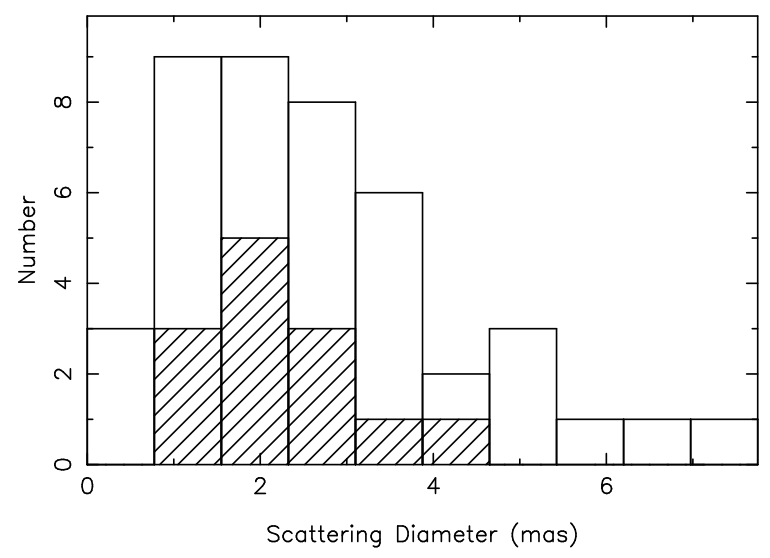

Figure 4: Histograms of scattering diameters. The open histogram shows the distribution for the scintillating sources; the hatched diagram shows the distribution for the non-scintillating sources.

Figure 4 shows a histogram of inferred angular diameters. A K-S test concludes that there is no difference in the angular diameters of the two populations: Scintillating and non-scintillating sources have the same distribution of scattering diameters with a typical value of $\sim 2$ mas at $1 \mathrm{GHz}$. Our favored explanation is that scintillation is produced from small "clumps" of scattering material, distributed throughout the Galactic disk, but which individually make no additional contribution to the angular broadening. In order to scintillate, a source must be compact enough and the line of sight to it must pass through a clump.

The distribution of scattering diameters as a function of redshift for the 37 sources with measured redshifts is shown in Figure 5. There is at best a marginal correlation (90\% confidence level) 
between scattering diameter and redshift, with scintillating (non-scintillating) sources becoming smaller (larger) at increasing redshift. As yet, there is no evidence of increasing scattering diameter at increasing redshift, as would be expected from intergalactic scattering.

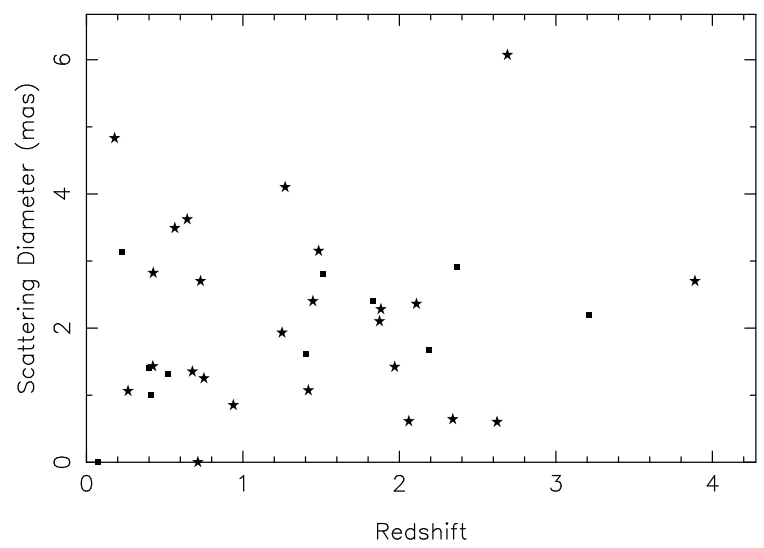

Figure 5: The distribution of scattering diameters as a function of redshift. Stars indicate sources that scintillate; squares indicate non-scintillating sources.

In conclusion, our multiwavelength observations and their analysis have shown that scattering is important for this sample of sources as a whole and measurable for most of them with a typical broadening diameter of 2 mas. Our results are consistent with the scenario of a highly turbulent IGM but we obtained no significant constraint on its properties.

\section{Implications for the improvement of the ICRF}

VLBI observations of selected strong compact extragalactic radio sources have been used to define and maintain a radio reference frame with sub-mas precision. This ICRF (International Celestial Reference Frame) was adopted as the fundamental celestial reference frame by the IAU in 1997 [7]. The ICRF is currently defined by the radio positions of 212 extragalactic objects obtained using the technique of VLBI at radio frequencies of 2.3 and $8.4 \mathrm{GHz}$ over the past $20+$ years.

The ICRF is currently limited by a deficit of defining sources, particularly in the Southern Hemisphere. Further, sources often have variable core-jet structures which cause position variations, though this can, in principle, be corrected. This latter problem is compounded by the fact that the ICRF is composed mostly of the brighter $(>0.2 \mathrm{Jy}$ at $8.4 \mathrm{GHz}$ ) sources many of which suffer the most from structure problems. Thus, future improvement of the ICRF will involve increasing the number of defining sources and by incorporating sources that have little or no structure, presumably leading to increased position stability.

Scintillating sources have proved to be some of the most compact sources known. This may make them ideal reference sources for the next generation (Mk IV/V) astrometry and geodesy reference frames. The increased sensitivity of Mk IV/V VLBI will probably be required to observe the generally weaker IDV sources. While the compact morphology of IDV sources suggests their use as ICRF sources, their position stability will need to be established; scintillation might provide the ultimate limitation for VLBI astrometry. 


\section{Summary}

Comparison of the morphologies of scintillators and non-scintillators clearly show that the former are significantly more core-dominated and have smaller angular extent. This could both be a limit for VLBI astrometry as well as a means of selecting good candidates for future realizations of the ICRF.

Multifrequency observations of scintillating and non-scintillating AGN have found scattering to be important and have allowed us to investigate a number of effects but have not, so far, allowed us to put any significant constraints on the IGM.

\section{References}

[1] H. Bignall et al., Rapid Interstellar Scintillation of PKS 1257-386: Two-Station Pattern Time Delays and Constraints on Scattering and Microarcsecond Source Structure, ApJ, 652, 1050-1058 (2006)

[2] J. Dennett-Thorpe et al., Interstellar scintillation as the origin of the rapid radio variability of the quasar J1819+3845, Nature, 415, 57-60 (2002)

[3] D. Jauncey et al., Interstellar Scintillation and Annual Cycles in the BL Lac Source PKS 1519-273, Ap\&SS, 288, 63-68 (2003)

[4] T. J. W. Lazio, R. Ojha, A. L. Fey, L. Kedziora-Chudczer, J. M. Cordes, D. L. Jauncey and J. E. J. Lovell, Angular Broadening of Intraday Variable AGN. II. Interstellar and Intergalactic Scattering, ApJ, 672, 115 (2008) [arXiv:0707.1778]

[5] J. E. J. Lovell, D. L. Jauncey, C. Senkbeil, S. Shabala, H. E. Bignall, T. Pursimo, R. Ojha, J. -P. Macquart, B. J. Rickett, M. S. Dutka and L. Kedziora-Chudczer, MASIV: The Microarcsecond Scintillation-Induced Variability Survey, ASPC, 365, 279-290 (2007)

[6] J. E. J. Lovell, D. L. Jauncey, H. E. Bignall, L. Kedziora-Chudczer, J. -P. Macquart, B. J. Rickett, A. K. Tzioumis, First Results from MASIV: The Microarcsecond Scintillation-induced Variability Survey, AJ, 126, 1699-1706 (2003)

[7] C. Ma et al., The International Celestial Reference Frame as Realized by Very Long Baseline Interferometry, AJ, 116, 516-546 (1998)

[8] R. Ojha, A. L. Fey, J. E. J. Lovell, D. L. Jauncey and K. J. Johnston, VLBA Snapshot Imaging Survey of Scintillating Sources, AJ, 128, 1570-1587 (2004)

[9] R. Ojha, A. L. Fey, D. L. Jauncey, J. E. J. Lovell, and K. J. Johnston, Milliarcsecond Structure of Microarcsecond Sources:Comparison of Scintillating and Nonscintillating Extragalactic Radio Sources, ApJ, 614, 607-614 (2004)

[10] R. Ojha, A. L. Fey, T. J. W. Lazio, D. L. Jauncey, J. E. J. Lovell, and L. Kedziora-Chudczer, Scatter Broadening of Scintillating and Nonscintillating AGNs. I. A Multifrequency VLBA Survey, ApJS, 166, 37-68 (2006)

[11] B. J. Rickett, Radio Propagation through the turbulent interstellar plasma, ARA\&A, 28, 561-605 (1990)

[12] S. J. Wagner and A. Witzel, Intraday Variability In Quasars and BL Lac Objects, ARA\&A, 33, 163-198 (1995) 